\section{PROLIFERATING CELL NUCLEAR ANTIGEN ANTIBODY TESTING - GETTING THEM ALL}

Christine Bundell $^{1,2}$, Mathew Krummenacher ${ }^{1,3}$, Elina Tan ${ }^{1,3}$, Paul Sjollema $^{4}$, Nick Acquarola ${ }^{4}$, Una Whitesmith ${ }^{1}$, Anna Brusch ${ }^{1,3}$

${ }^{1}$ PathWest Laboratory Medicine, QEII Medical Centre, Nedlands, WA, Australia; ${ }^{2}$ School of Biomedical Sciences, University of Western Australia, Crawley, WA, Australia; ${ }^{3}$ Clinical Immunology, Sir Charles Gairdner Hospital, QEII Medical Centre, Nedlands, WA, Australia; and ${ }^{4}$ PathWest Laboratory Medicine, Fiona Stanley Hospital, Murdoch, WA, Australia

Proliferating cell nuclear antigen antibody (PCNA) is associated with systemic lupus erythematosus (SLE) and characterises a cohort of patients at risk of renal or central nervous system involvement. The antibody has also been reported in patients with hepatitis $\mathrm{B}$ and $\mathrm{C}$ virus infection. It has a pleomorphic nuclear cell cycle dependant pattern on antinuclear antibody (ANA) testing by indirect immunofluorescence (IFA).

In this laboratory PCNA has been historically reported from the IFA pattern on Hep2000 cells (Immuno Concepts, USA) and not confirmed on a secondary test.

An audit of PCNA pattern reported from ANA testing and incidental characterisation by immunoblot was undertaken to determine the frequency of PCNA detected. Over 4 years 34 PCNA results were reported. Clinical details provided included SLE, joint pain and acute hepatitis.

Extractable nuclear antigen antibody characterisation by immunoblot incidentally identified a further 20 samples with PCNA not reported on ANA. ANA patterns included speckled, homogeneous and nucleolar. Clinical details in this group also included SLE and joint pain.

This investigation demonstrates that IFA testing alone for PCNA may miss clinically relevant cases due to a strong dominant ANA pattern. Clinical characteristics and correlation with IFA is required to validate the immunoblot PCNA result.

\section{ANALYTICAL COMPARISON OF A PD-L1 22C3 ANTIBODY LABORATORY-DEVELOPED TEST (LDT) PROTOCOL ON THE BENCHMARK XT AND PD-L1 IHC 22C3 PHARMDX: ANALYSIS OF PAN-TUMOUR AND OESOPHAGEAL CANCER SAMPLES}

Gilad W. Vainer ${ }^{1}$, Ghadeer Zatara ${ }^{1}$, Lingkang Huang ${ }^{2}$, Kenneth Emancipator ${ }^{2}$, Shanthy Nuti ${ }^{2}$

${ }^{1}$ Hadassah-Hebrew University Medical Center, Jerusalem, Israel; and ${ }^{2}$ Merck \& Co Inc, Kenilworth, New Jersey, USA

PD-L1 IHC 22C3 pharmDx is an FDA-approved companion diagnostic to pembrolizumab designed for use on Autostainer Link 48. We compared our PD-L1 22C3 antibody-based LDT ${ }^{1}$ on BenchMark XT with PD-L1 IHC 22C3 pharmDx. Head and neck squamous cell carcinoma (HNSCC), urothelial carcinoma (UC), and oesophageal SCC (ESCC) tumour specimens were stained with 22C3 antibody, scored using the LDT on BenchMark XT, and compared with PD-L1 IHC 22C3 pharmDx. PDL1 was measured using combined positive score (CPS) and standard cutoffs (HNSCC, $\geq 1$; UC, $\geq 10$; ESCC, $\geq 10$ ). 327 samples were evaluated (HNSCC, $n=126$; UC, $n=121$; ESCC, $n=80$ ). Pan-tumour intraclass correlation coefficient (ICC) of
PD-L1 CPS as a continuous variable was 0.96 (95\% CI, 0.95-0.97); Spearman correlation was 0.95. For ESCC, ICC was 0.92 (95\% CI, 0.88-0.95); Spearman correlation was 0.89 . Interpretation of PD-L1 status (CPS $\geq 10$ ) for ESCC using the two assays resulted in NPA of $92 \%$ (95\% CI, 80-97), PPA of $88 \%$ (95\% CI, 74-95), and OPA of 90\% (95\% CI, 81-95). 22C3 antibody-based LDT on BenchMark XT demonstrated high concordance with PD-L1 IHC 22C3 pharmDx. These findings suggest comparability of PD-L1 IHC 22C3 pharmDx with an LDT based on the $22 \mathrm{C} 3$ antibody across several tumour types. Reference

1. Neuman T, London M, Kania-Almog J, et al. A harmonization study for the use of 22C3 PD-L1 immunohistochemical staining on Ventana's platform. J Thorac Oncol 2016; 11: 1863-8.

\section{CONTRIBUTION OF TUMOUR AND IMMUNE CELLS TO PD-L1 AS A PREDICTIVE BIOMARKER IN METASTATIC TRIPLE-NEGATIVE BREAST CANCER (MTNBC): ANALYSIS FROM KEYNOTE-119}

Kenneth Emancipator $^{1}$, Eric P. Winer ${ }^{2}$, Oleg Lipatov ${ }^{3}$, Seock-Ah Im ${ }^{4}$, Anthony Goncalves ${ }^{5}$, Eva Muñoz-Couselo ${ }^{6}$, Keun Seok Lee ${ }^{7}$, Zbigniew Nowecki ${ }^{8}$, Peter Schmid ${ }^{9}$, Kenji Tamura ${ }^{10}$, Laura Testa ${ }^{11}$, Isabell Witzel ${ }^{12}$, Shoichiro Ohtani ${ }^{13}$, Stephanie Hund ${ }^{14}$, Karina Kulangara ${ }^{14}$, Vassiliki Karantza ${ }^{1}$, Jaime A. Mejia ${ }^{1}$, Junshui $\mathrm{Ma}^{1}$, Petar Jelinic $^{1}$, Lingkang Huang ${ }^{1}$, Javier Cortes ${ }^{15,16}$ ${ }^{1}$ Merck \& Co. Inc., Kenilworth, NJ, USA; ${ }^{2}$ Dana-Farber Cancer Institute, Harvard Medical School, Boston, MA, USA, ${ }^{3}$ Republican Clinical Oncology Dispensary of the Ministry of Public Health of Bashkortostan Republic, Ufa, Russian Federation; ${ }^{4}$ Seoul National University College of Medicine, Seoul National University Hospital, and Cancer Research Institute, Seoul National University, Seoul, Republic of Korea; ${ }^{5}$ Institut Paoli-Calmettes, Marseille, France; ${ }^{6}$ Vall d'Hebron University Hospital, Barcelona, Spain; ${ }^{7}$ Center for Breast Cancer, Research Institute and Hospital, National Cancer Center, Goyang, Republic of Korea; ${ }^{8}$ Breast Cancer and Reconstructive Surgery Clinic, Center of Oncology Institute, Warsaw, Poland; ${ }^{9}$ Barts ECMC, Barts Cancer Institute, Queen Mary University of London, and Barts Health NHS Trust, London, United Kingdom; ${ }^{10}$ National Cancer Center Hospital, Tokyo, Japan; ${ }^{11}$ Instituto do Câncer do Estado de São Paulo, Faculdade de Medicina da Universidade de São Paulo, São Paulo, Brazil; ${ }^{12}$ University Medical Center HamburgEppendorf, Hamburg, Germany; ${ }^{13}$ Hiroshima City Hiroshima Citizens Hospital, Hiroshima, Japan; ${ }^{14}$ Agilent Technologies, Carpinteria, CA, USA; ${ }^{15}$ IOB Institute of Oncology, Quiron Group, Madrid and Barcelona, Spain; and ${ }^{16}$ Vall d'Hebron Institute of Oncology (VHIO), Barcelona, Spain

Background: We evaluated whether PD-L1 expression on tumour cells contributes to PD-L1 as a predictive biomarker for pembrolizumab in mTNBC.

Methods: Patients with $\mathrm{mTNBC}$ received pembrolizumab or chemotherapy. PD-L1 expression (PD-L1 IHC 22C3 pharmDx) was quantified per tumour proportion score (TPS; percentage of PD-L1-expressing tumour cells [partial or complete membrane staining] relative to total number of tumour cells) and combined positive score (CPS; number of PD-L1-staining cells [tumour cells, lymphocytes, macrophages] divided by total number of viable tumour cells, multiplied by 100). Quantitative immune cell 